\title{
Identification of yellow rust resistance sources in advanced breeding lines of barley (Hordeum vulgare $\mathrm{L}$. )
}

Jogendra Singh*1, Om Prakash Gangwar ${ }^{2}$, Sudheer Kumar ${ }^{1}$, Pradeep Singh Shekhawat ${ }^{3}$, Dinesh Kumar ${ }^{1}$, Vishnu Kumar ${ }^{1}$, Jaspal Kaur ${ }^{4}$, Mukesh Kumar Pandey ${ }^{5}$, Rakesh Devlash ${ }^{6}$, Ajeet Singh Kharub ${ }^{1}$ and Gynendra Pratap Singh ${ }^{1}$

${ }^{1}$ ICAR-Indian Institute of Wheat and Barley Research, Karnal-132 001, Haryana, India

${ }^{2}$ ICAR-Indian Institute of Wheat and Barley Research, Regional Station, Flowerdale, Shimla-171 002, Himachal Pradesh, India

${ }^{3}$ SKNAU-Rajasthan Agricultural Research Institute, Durgapura-302 018, Rajasthan, India

${ }^{4}$ Department of Plant Breeding and Genetics, Punjab Agricultural University, Ludhiana-141004, India

${ }^{5}$ SKUAST-J., Main Campus, Chatha, Jammu-180 009, JEK, India

${ }^{6}$ CSK HPKVV, Hill Agricultural Research and Extension Centre, Bajaura-175 125, Kulu, Himachal Pradesh, India

\section{Article history}

Received: 16 Jun., 2019

Revised : 12 Aug., 2019

Accepted: 29 Aug., 2019

\section{Citation}

Singh J, OP Gangwar, S Kumar, PS Shekhawat, D Kumar, V Kumar, J Kaur, MK Pandey, R Devlash, AS Kharub and GP Singh 2019. Identification of yellow rust resistance sources in advanced breeding lines of barley (Hordeum vulgare L.) Journal of Cereal Research 11(2): 160-165 doi.org10.25174/2249-4065/2019/85076

\section{*Corresponding author}

Email: jogendrasail@yahoo.co.in,

\begin{abstract}
Yellow rust of barley caused by Puccinia striiformis f. sp. hordei (Psh), is an important disease in barley growing regions of India and worldwide. For identifying adult plant stage resistance to yellow rust, a set of 29 barley advanced breeding lines was tested at five locations, Durgapura, Jammu, Ludhiana, Bajaura and Karnal during 2016-17 and 2017-18. The seedling resistance test was conducted on 27 barley advanced breeding lines against seven $P S h$ pathotypes separately under controlled conditions at ICAR-IIWBR, Shimla during 2017-18. As consequences, seven lines (DWRFB10, DWRFB12, DWRFB14, DWRFB15, DWRFB19, DWRFB20 and DWRFB28) were found immune to highly resistant against yellow rust at adult plant stage across the locations. Similarly, seven advanced breeding lines viz, DWRFB11, DWRFB12, DWRFB13, DWRFB14, DWRFB19, DWRFB20 and DWRFB27, were found resistant to yellow rust at seedling stage. On the basis of APR and SRT, four advanced barley breeding lines, DWRFB12, DWRFB14, DWRFB19 and DWRFB20 were highly resistant to yellow rust (nearly immune) both at seedling and adult-plant stages.
\end{abstract}

Keywords: Hordeum vulgare, barley, yellow rust, barley diseases, genetic resistance, Puccinia striiformis

\section{Introduction}

Barley (Hordeum vugare L.) is one of the founder crops of the world agriculture. According to the acreage and production, barley occupied fourth ranks after wheat, rice and maize at world level during 2009 (Pandey et al., 2009). It is cultivated in varying agro-climatic conditions such as rainfed areas, dry lands, salinealkaline soils, flood prone, marginal and coastal areas in the world. Hence, barley is considered an important crop for resource poor farmers in many developing countries. In India, barley is grown about 0.693 million hectares area with 1.79 million tonnes production and a productivity of $2580 \mathrm{~kg} / \mathrm{ha}$ (Anonymous, 2017). It is consumed in varied forms including animal feed, human food and for malting and brewing in industry. This crop is considered as poor man's crop in India because of its low input requirement and better adaptability in the harsh environments (Verma et al., 2012).

Barley suffers from several diseases responsible for heavy reduction in yield and grain quality. Out of them, yellow rust is an important disease of barley, caused by Puccinia striiformis Westend. f. sp. hordei Eriks. \& Henn. (Psh). Generally, it occurs in the barley growing areas of the northern India and cooler parts of many countries of world (Prashar et al., 2014).The disease initiates appearing in the plains during midDecember to beginning of January and thrives well 
under the cold conditions (Prakash and Verma, 2009). It is also prevalent and destructive at higher altitude in Ladakh region of India (Vaish et al., 2011). The incidence of yellow rust may create serious problems by growing susceptible varieties and as a consequence in heavy yield losses. Yield losses caused by P. striiformis f. sp. hordei were estimated up to 60\% (Park et al., 2007). Severe epidemics of the disease have been observed in north-western and central European countries, India, Bangladesh, Nepal, China and Japan (Chen et al., 1995). Early incidence of yellow rust disease can cause heavy damage to barley crop in Indian environmental conditions and sometimes, it prevents emergence of heads or grain formation (Prakash and Verma, 2009). The yield losses may be reduced by controlling the disease through application of the fungicides (Marshall and Sutton, 1995). However, it is not considered as an economical and environmental friendly approach to control the disease. Therefore, development of resistant varieties against yellow rust becomes inevitable. The current study was conducted to identify the resistant sources to yellow rust disease in twenty nine advanced breeding lines at adult-plant stage and in twenty seven lines at seedling stage.

\section{Materials and methods}

\subsection{Adult Plant Resistance Test}

The experimental material comprised of total 29 advanced breeding lines of feed barley which were evaluated against yellow rust disease at five different hot spot locations (Durgapura, Ludhiana, Bajaura, Jammu and Karnal) during 2016-17 and 2017-18 seasons under artificial rust epiphytotic conditions (Table 1). The field experiments were conducted in the month of November of both consecutive years. Each test line was sown in single row of $1.0 \mathrm{~m}$ length and a distance of $30 \mathrm{~cm}$ was maintained between the rows. A susceptible check (Bilara-2) was raised before the first and after the last test lines. Susceptible infector lines (mixture of susceptible cultivars BL2, RD31, RS6, Jyoti and RD2035) were sown on all four sides of the test lines. The susceptible infector rows were artificially injected with the spore suspension prepared by mixing of uredospores of five Psh pathotypes i.e. 24(0S0-1), 57(0S0), G (4S0), M (1S0) \& Q (5S0), just before early tillering stage (Zadoks GS 10-19). Additionally, in the main field, infectors were also inoculated 3-4 times by spraying from tillering to flag leaf stage to trigger the development of rust epidemics. Five irrigations were applied at different growth stages to the crop for maintaining the moisture and disease development. The observations on yellow rust were recorded by combining severity (percent leaf area covered by rust) and response (infection type). The plants were scored when the disease appeared completely on the infector rows. The scoring for yellow rust was done using the modified Cobb's scale (Peterson et al., 1948). The host response in the field was scored as Resistant $(\mathrm{R})=$ no uredia present; Moderately Resistant $(\mathrm{MR})=$ small uredia with slight sporulation; Moderately Susceptible $(\mathrm{MS})=$ medium sized uredia with moderate to heavy sporulation and Susceptibility $(\mathrm{S})=$ large uredia with abundant sporulation. The disease severity and host response data were combined into a single value

Table1. Advanced breeding lines of barley derived by crossing between indigenous and exotic genotypes for APR and SRT study

\begin{tabular}{llll}
\hline \multicolumn{1}{c}{ Genotype } & \multicolumn{1}{c}{ Pedigree } & \multicolumn{1}{c}{ Genotype } & \multicolumn{1}{c}{ Pedigree } \\
\hline DWRFB1 & RD 2715/BCU 8 & DWRFB16 & IBYT-HI-9 (2010-11)/BCU 6631 \\
DWRFB2 & RD 2715/BCU 8 & DWRFB17 & IBYT-HI-1 (2010-11)/BCU 2881 \\
DWRFB3 & RD 2715/BCU 1549 & DWRFB18 & IBYT-HI-1 (2010-11)/BCU2881 \\
DWRFB4 & RD 2715/BCU 1549 & DWRFB19 & CDC MANLEY/BCU 2881 \\
DWRFB5 & P L 426/EIBON 18 & DWRFB20 & CDC MANLEY/BCU 2881 \\
DWRFB6 & P L 426/EIBON 19 & DWRFB21 & RD 2052/RD 2092 \\
DWRFB7 & PL 426/EIBON 19 & DWRFB22 & DL 456/RD 2592 \\
DWRFB8 & PL 426/EIBON 19 & DWRFB23 & RD 2035/BCU 6038 \\
DWRFB9 & P L 426/IBYT-LRA-C1 & DWRFB24 & VM 150/BCU 8 \\
DWRFB10 & DWR 83/EIBON 18 & DWRFB25 & VM 150/BCU 8 \\
DWRFB11 & DWR 83/EIBON 18 & DWRFB26 & VM 150/BH 902 \\
DWRFB12 & DWR 83/EIBON 18 & DWRFB27 & RD 2552/IND 253 \\
DWRFB13 & DWR 83/EIBON 18 & DWRFB28 & IBON-(2015)-59 \\
DWRFB14 & DWR 83/EIBON 19 & DWRFB29 & IBON-(2015)-49 \\
DWRFB15 & DWR 83/EIBON 19 & & \\
\hline
\end{tabular}


called the coefficient of infection (CI). The coefficient of infection was estimated by multiplying of disease severity (DS) and constant values of infection type (IF). The constant values for infection types were immune $=0.0, \mathrm{R}=0.2, \mathrm{MR}=0.4, \mathrm{M}=0.6, \mathrm{MS}=0.8$ and $\mathrm{S}=1.0$ (Stubbs et al., 1986). The average coefficient of infection (ACI) was calculated. The categorization of advanced lines into resistance and susceptible was done based on ACI values (Sajid et al., 2009). The advanced lines with pooled ACI value 0 or $<1$, were considered highly resistant (nearly immune). The lines with ACI values of 0-5 were considered to possess strong seedling resistance instead of adult-plant resistance. However, ACI values 5-20 were considered as high adult-plant resistance, 21- 40 as moderate and 41-60 as low adultplant resistance. Advanced lines with ACI values $>60$ were considered susceptible or no adult-plant resistance.

\subsection{Seedling resistance test}

The seedling resistance test was performed on twenty seven barley advanced breeding lines during 201718 at Regional Station, IIWBR, Flowerdale, Shimla, using seven pathotypes (6S0, 7S0, G, M, 24, 57 and Q) separately under controlled conditions. Barley lines were grown in aluminium bread pans $(29 \times 12$ x $7 \mathrm{~cm}$ ) containing a mixture of fine loam and farm yard manure (3:1). These trays were sufficiently large to accommodate 18 lines and a susceptible check (Bilara-2). For each barley line, about 4-5 seeds were sown in hills. One-week old seedlings were inoculated using a glass atomizer containing $10 \mathrm{mg}$ spores of an individual Psh pathotype suspended in $1.0 \mathrm{ml}$ light grade mineral oil (Soltrol $170^{\circledR}$ ). Thereafter, inoculated barley lines were sprayed with a fine mist of water and kept in a moist chamber $(\mathrm{RH}>80 \%)$ at $12 \pm 2^{\circ} \mathrm{C}$ for 48 hours. Subsequently, they were transferred on to the green house benches where appropriate temperature $\left(16 \pm 2^{\circ} \mathrm{C}\right)$, relative humidity $(60-80 \%)$ and illumination (about 15,000 lx for 12 hours) were maintained (Gangwar et al., 2018). The data were recorded on reaction type of these lines against each pathotype at 16-18 days post-inoculation (Nayar et al., 1997).

\section{Results and discussion}

Yellow rust is one of the major biotic stresses in barley production. Yellow rust pathogen is obligate parasite of crop plants and evolves frequently in many distinct physiologic races or pathotypes. New pathotypes spread rapidly because of high reproductive rate and airborne nature (Duveiller et al., 2007; Duplessis et al., 2011). Pathotype $6 \mathrm{~S} 0$ and $7 \mathrm{~S} 0$ are newly emerged and virulent on both wheat and barley hosts. Pathotype $1 \mathrm{~S} 0(\mathrm{M})$ is predominant and widely distributed across the North Indian states followed by pathotype 0S0 (57) (Prashar et al., 2014; Gangwar et al., 2016, Gangwar et al., 2019).
The major resistance genes are generally overcome by new virulent pathotypes and thus, identification and exploitation of new sources of resistance has become essential for sustainable rust resistance breeding program. In this study, seven advanced breeding lines (DWRFB11, DWRFB12, DWRFB13, DWRFB14, DWRFB19, DWRFB20 and DWRFB27) were found resistant (IT: 0;) to all Psh pathotypes at seedling stage. On the contrary, 12 advanced breeding lines were susceptible (IT: $3,3^{+}$) to all the pathotypes. The infection types (ITs) on advanced breeding lines at seedling stage (all-stage resistance) are presented in Table 2. The identified resistant sources to yellow rust

Table 2. Seedling Resistance Test (SRT) of advanced breeding lines of barley to individual race of Puccinia striiformis f.sp. hordei

\begin{tabular}{|c|c|c|c|c|c|c|c|}
\hline \multirow[t]{2}{*}{ Genotype } & \multicolumn{7}{|c|}{ Reaction" to $P s h$ races } \\
\hline & $6 \mathrm{SO}$ & $7 \mathrm{~S} 0$ & G & M & 24 & 57 & Q \\
\hline DWRFB1 & $33^{+}$ & $3^{+}$ & $3^{+}$ & $3^{+}$ & $3^{+}$ & $3^{+}$ & $3^{+}$ \\
\hline DWRFB2 & $3^{+}$ & $3^{+}$ & $3^{+}$ & $3^{+}$ & $3^{+}$ & $3^{+}$ & $3^{+}$ \\
\hline DWRFB3 & $3^{+}$ & $33^{+}$ & 3 & $2^{+}$ & $3^{+}$ & $3^{+}$ & 2 \\
\hline DWRFB4 & $3^{+}$ & $3^{+}$ & 3 & $3^{+}$ & $3^{+}$ & $3^{+}$ & 2 \\
\hline DWRFB5 & $3^{+}$ & $3^{+}$ & $3^{+}$ & $3^{+}$ & $3^{+}$ & $3^{+}$ & 3 \\
\hline DWRFB6 & $3^{+}$ & $3^{+}$ & $3^{+}$ & $3^{+}$ & $3^{+}$ & $3^{+}$ & 3 \\
\hline DWRFB7 & $3^{+}$ & $3^{+}$ & $3^{+}$ & $3^{+}$ & $3^{+}$ & $3^{+}$ & $3^{+}$ \\
\hline DWRFB8 & $3^{+}$ & $3^{+}$ & $3^{+}$ & $3^{+}$ & $3^{+}$ & $3^{+}$ & $3^{+}$ \\
\hline DWRFB9 & $33^{+}$ & $3^{+}$ & $3^{+}$ & $3^{+}$ & $3^{+}$ & $3^{+}$ & 0 \\
\hline DWRFB 10 & $3^{+}$ & $3^{+}$ & $3^{+}$ & $3^{+}$ & $3^{+}$ & $3^{+}$ & $3^{+}$ \\
\hline DWRFB11 & 0 & 0 & 0 & 0 & 0 & 0 & 0 \\
\hline DWRFB12 & 0 & 0 & 0 & 0 & 0 & 0 & 0 \\
\hline DWRFB 13 & 0 & 0 & 0 & 0 & 0 ; & 0 & 0 \\
\hline DWRFB14 & 0 & 0 & 0 & 0 & 0 & 0 & 0 \\
\hline DWRFB 15 & 0 & $3^{+}$ & 0 & 0 ; & 0 & 0 & 0 \\
\hline DWRFB 16 & $3^{+}$ & $3^{+}$ & $3^{+}$ & $3^{+}$ & $3^{+}$ & $3^{+}$ & $3^{+}$ \\
\hline DWRFB 17 & 3 & 0 & 2 & 0 & 3 & 2 & 0 \\
\hline DWRFB18 & 3 & 3 & 2 & 0 & 0 & 3 & 0 \\
\hline DWRFB19 & 0 & 0 & 0 & 0 & 0 & 0 & 0 \\
\hline DWRFB20 & 0 & 0 & 0 & 0 & 0 & 0 & 0 \\
\hline DWRFB21 & $3^{+}$ & $3^{+}$ & $3^{+}$ & $3^{+}$ & $3^{+}$ & $3^{+}$ & $3^{+}$ \\
\hline DWRFB22 & 3 & $3^{+}$ & $3^{+}$ & $3^{+}$ & $3^{+}$ & $3^{+}$ & $3^{+}$ \\
\hline DWRFB23 & $33^{+}$ & 3 & $3^{+}$ & 3 & $3^{+}$ & $33^{+}$ & 2 \\
\hline DWRFB24 & $3^{+}$ & $3^{+}$ & $3^{+}$ & $3^{+}$ & $3^{+}$ & $3^{+}$ & 2 \\
\hline DWRFB25 & $3^{+}$ & 3 & $3^{+}$ & 3 & $3^{+}$ & $3^{+}$ & 3 \\
\hline DWRFB26 & $3^{+}$ & $3^{+}$ & $3^{+}$ & $3^{+}$ & $3^{+}$ & $3^{+}$ & $3^{+}$ \\
\hline DWRFB27 & 0 & 0 & 0 & 0 ; & 0 & 0 & 0 \\
\hline BHS $352(\mathrm{C})$ & 3 & - & 3 & $3^{+}$ & 3 & $3^{+}$ & 2 \\
\hline Gitanjali (c) & $3^{+}$ & $3^{+}$ & $3^{+}$ & $3^{+}$ & $3^{+}$ & $3^{+}$ & $3^{+}$ \\
\hline Karan 16 & $3^{+}$ & $3^{+}$ & $3^{+}$ & $3^{+}$ & $3^{+}$ & $3^{+}$ & $3^{+}$ \\
\hline NDB 943 & $3^{+}$ & $3^{+}$ & $3^{+}$ & $3^{+}$ & $3^{+}$ & $3^{+}$ & $3^{+}$ \\
\hline
\end{tabular}
resistant, $0 ; / ; / 1 / 2=$ Resistant 
Table3. Field Response of advanced barley breeding lines to yellow rust reactions

\begin{tabular}{|c|c|c|c|c|c|c|c|c|c|c|c|c|c|}
\hline \multirow[b]{2}{*}{ Genotype } & \multicolumn{6}{|c|}{ IBDSN 2016-17 } & \multicolumn{6}{|c|}{ IBDSN 2017-18 } & \multirow[b]{2}{*}{$\begin{array}{l}\text { Pooled } \\
\text { ACI value }\end{array}$} \\
\hline & 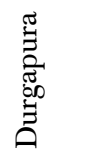 & 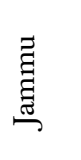 & 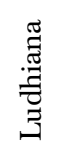 & 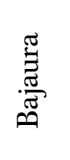 & 苂 & 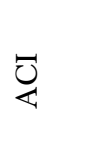 & 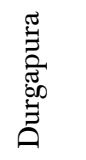 & 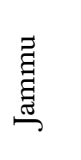 & 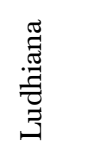 & 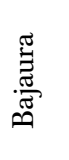 & 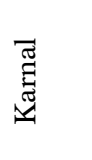 & 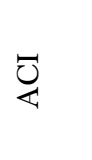 & \\
\hline DWRFB1 & $100 \mathrm{~S}$ & $40 \mathrm{~S}$ & $5 \mathrm{~S}$ & $80 \mathrm{~S}$ & $60 \mathrm{~S}$ & 57.00 & $80 \mathrm{~S}$ & $10 \mathrm{~S}$ & $20 \mathrm{~S}$ & $60 \mathrm{~S}$ & $40 \mathrm{~S}$ & 42.00 & 49.50 \\
\hline DWRFB2 & $80 \mathrm{~S}$ & $40 \mathrm{~S}$ & $10 \mathrm{~S}$ & $80 \mathrm{~S}$ & $60 \mathrm{~S}$ & 54.00 & $80 \mathrm{~S}$ & $10 \mathrm{~S}$ & $20 \mathrm{~S}$ & $60 \mathrm{~S}$ & $40 \mathrm{~S}$ & 42.00 & 48.00 \\
\hline DWRFB3 & $30 \mathrm{MS}$ & $10 \mathrm{~S}$ & 0 & $80 \mathrm{~S}$ & 0 & 22.80 & $20 \mathrm{~S}$ & 0 & $10 \mathrm{~S}$ & $60 \mathrm{~S}$ & $5 \mathrm{MR}$ & 18.40 & 20.60 \\
\hline DWRFB4 & $40 \mathrm{~S}$ & 0 & 0 & $80 \mathrm{~S}$ & $10 \mathrm{~S}$ & 26.00 & $40 \mathrm{~S}$ & 0 & $20 \mathrm{~S}$ & $60 \mathrm{~S}$ & $10 \mathrm{~S}$ & 26.00 & 26.00 \\
\hline DWRFB5 & $60 \mathrm{~S}$ & $40 \mathrm{~S}$ & $5 \mathrm{~S}$ & $60 \mathrm{~S}$ & $40 \mathrm{~S}$ & 41.00 & $60 \mathrm{~S}$ & $10 \mathrm{~S}$ & $40 \mathrm{~S}$ & $60 \mathrm{~S}$ & $10 \mathrm{MS}$ & 35.60 & 38.30 \\
\hline DWRFB6 & $60 \mathrm{~S}$ & $20 \mathrm{~S}$ & $5 \mathrm{~S}$ & $60 \mathrm{~S}$ & $40 \mathrm{~S}$ & 37.00 & $40 \mathrm{~S}$ & $5 \mathrm{R}$ & $40 \mathrm{~S}$ & $40 \mathrm{~S}$ & $20 \mathrm{~S}$ & 28.20 & 32.60 \\
\hline DWRFB7 & $60 \mathrm{~S}$ & $20 \mathrm{~S}$ & $5 \mathrm{~S}$ & $60 \mathrm{~S}$ & $40 \mathrm{~S}$ & 37.00 & $40 \mathrm{~S}$ & 0 & $20 \mathrm{~S}$ & $60 \mathrm{~S}$ & $20 \mathrm{~S}$ & 28.00 & 32.50 \\
\hline DWRFB8 & $30 \mathrm{~S}$ & $20 \mathrm{~S}$ & $5 \mathrm{~S}$ & 0 & $60 \mathrm{~S}$ & 23.00 & $20 \mathrm{~S}$ & $5 \mathrm{R}$ & $20 \mathrm{~S}$ & $40 \mathrm{~S}$ & $20 \mathrm{~S}$ & 20.20 & 21.60 \\
\hline DWRFB9 & $60 \mathrm{~S}$ & $5 \mathrm{MS}$ & $10 \mathrm{~S}$ & $80 \mathrm{~S}$ & $40 \mathrm{~S}$ & 38.80 & $80 \mathrm{~S}$ & $20 \mathrm{~S}$ & $40 \mathrm{~S}$ & $30 \mathrm{~S}$ & $20 \mathrm{~S}$ & 38.00 & 38.40 \\
\hline DWRFB10 & 0 & 0 & 0 & 0 & $20 \mathrm{~S}$ & 4.00 & 0 & TMS & 0 & 0 & 0 & 0.16 & 2.08 \\
\hline DWRFB11 & 0 & 0 & 0 & $80 \mathrm{~S}$ & 0 & 16.00 & 0 & 0 & 0 & 0 & 0 & 0.00 & 8.00 \\
\hline DWRFB12 & 0 & $5 \mathrm{MS}$ & 0 & 0 & 0 & 0.80 & 0 & 0 & 0 & 0 & 0 & 0.00 & 0.40 \\
\hline DWRFB 13 & 0 & $5 \mathrm{MS}$ & 0 & $60 \mathrm{~S}$ & 0 & 12.80 & 0 & $10 \mathrm{~S}$ & 0 & 0 & 0 & 2.00 & 7.40 \\
\hline DWRFB14 & 0 & 0 & 0 & 0 & 0 & 0.00 & 0 & 0 & 0 & 0 & 0 & 0.00 & 0.00 \\
\hline DWRFB15 & 0 & TMS & 0 & 0 & 0 & 0.16 & 0 & 0 & 0 & 0 & $20 \mathrm{~S}$ & 4.00 & 2.08 \\
\hline DWRFB16 & $80 \mathrm{~S}$ & $5 \mathrm{~S}$ & $5 \mathrm{~S}$ & $80 \mathrm{~S}$ & $40 \mathrm{~S}$ & 42.00 & $80 \mathrm{~S}$ & 0 & $40 \mathrm{~S}$ & $60 \mathrm{~S}$ & $40 \mathrm{~S}$ & 44.00 & 43.00 \\
\hline DWRFB17 & 0 & $20 \mathrm{~S}$ & 0 & $60 \mathrm{~S}$ & 0 & 16.00 & $5 \mathrm{R}$ & $20 \mathrm{~S}$ & $5 \mathrm{MS}$ & $40 \mathrm{~S}$ & 0 & 13.00 & 14.50 \\
\hline DWRFB18 & $5 \mathrm{MS}$ & $5 \mathrm{MS}$ & 0 & $80 \mathrm{~S}$ & 0 & 17.60 & $10 \mathrm{~S}$ & 0 & $5 \mathrm{~S}$ & $40 \mathrm{~S}$ & $5 \mathrm{MR}$ & 11.40 & 14.50 \\
\hline DWRFB19 & 0 & 0 & 0 & 0 & 0 & 0.00 & TR & $\mathrm{TR}$ & TR & 0 & 0 & 0.12 & 0.06 \\
\hline DWRFB20 & 0 & 0 & 0 & 0 & 0 & 0.00 & 0 & TR & 0 & 0 & 0 & 0.04 & 0.02 \\
\hline DWRFB21 & $100 \mathrm{~S}$ & $5 \mathrm{~S}$ & $20 \mathrm{~S}$ & $40 \mathrm{~S}$ & $40 \mathrm{~S}$ & 41.00 & $100 \mathrm{~S}$ & 0 & $10 \mathrm{~S}$ & 0 & $20 \mathrm{~S}$ & 26.00 & 33.50 \\
\hline DWRFB22 & $60 \mathrm{~S}$ & NIL & $10 \mathrm{~S}$ & $40 \mathrm{~S}$ & $40 \mathrm{~S}$ & 37.50 & $40 \mathrm{~S}$ & $20 \mathrm{~S}$ & $20 \mathrm{~S}$ & $40 \mathrm{~S}$ & $20 \mathrm{~S}$ & 28.00 & 32.75 \\
\hline DWRFB23 & $30 \mathrm{~S}$ & NIL & 0 & 0 & 0 & 7.50 & $20 \mathrm{~S}$ & $20 \mathrm{~S}$ & $10 \mathrm{~S}$ & $40 \mathrm{~S}$ & $10 \mathrm{MS}$ & 19.60 & 13.55 \\
\hline DWRFB24 & $60 \mathrm{~S}$ & NIL & $5 \mathrm{~S}$ & $40 \mathrm{~S}$ & $40 \mathrm{~S}$ & 36.25 & $20 \mathrm{~S}$ & 0 & $10 \mathrm{~S}$ & $60 \mathrm{~S}$ & $10 \mathrm{MS}$ & 19.60 & 27.92 \\
\hline DWRFB25 & $60 \mathrm{~S}$ & 0 & $5 \mathrm{~S}$ & $40 \mathrm{~S}$ & $20 \mathrm{~S}$ & 25.00 & $20 \mathrm{~S}$ & $20 \mathrm{~S}$ & $10 \mathrm{~S}$ & $60 \mathrm{~S}$ & $20 \mathrm{~S}$ & 26.00 & 25.50 \\
\hline DWRFB26 & $60 \mathrm{~S}$ & $10 \mathrm{~S}$ & $10 \mathrm{~S}$ & $60 \mathrm{~S}$ & $40 \mathrm{~S}$ & 36.00 & $20 \mathrm{~S}$ & 0 & $10 \mathrm{~S}$ & $60 \mathrm{~S}$ & $40 \mathrm{~S}$ & 26.00 & 31.00 \\
\hline DWRFB27 & 0 & $5 \mathrm{MS}$ & 0 & $20 \mathrm{~S}$ & 0 & 4.80 & 0 & TMS & 0 & $40 \mathrm{~S}$ & 0 & 8.16 & 6.48 \\
\hline DWRFB28 & $10 \mathrm{MS}$ & 0 & $5 \mathrm{~S}$ & 0 & 0 & 2.60 & 0 & TR & 0 & 0 & 0 & 0.04 & 1.32 \\
\hline DWRFB29 & $15 \mathrm{~S}$ & 0 & 0 & $15 \mathrm{~S}$ & $10 \mathrm{MS}$ & 7.60 & $10 \mathrm{~S}$ & TR & $5 \mathrm{~S}$ & 0 & $20 \mathrm{~S}$ & 7.04 & 7.32 \\
\hline Infector & $100 \mathrm{~S}$ & $60 \mathrm{~S}$ & $60 \mathrm{~S}$ & $80 \mathrm{~S}$ & $80 \mathrm{~S}$ & 76.0 & $100 \mathrm{~S}$ & $60 \mathrm{~S}$ & $60 \mathrm{~S}$ & $80 \mathrm{~S}$ & $60 \mathrm{~S}$ & 72.00 & 74.00 \\
\hline
\end{tabular}

IBDSN- Initial Barley Disease Screening Nursery; ACI- Average Coefficient of Infection; R- Resistant; TR-Traces to Resistant; MRModerately Resistant; TMR-Traces to Moderately Resistant; MS-Moderately Susceptible; S-Susceptible

in the present study can be exploited as donor parent in barley breeding program.

The adult plant resistance (APR) is usually expressed at adult plant stage and also referred as partial resistance, horizontal resistance, durable resistance and nonrace specific. Adult plant resistance is believed to be governed by several additive minor genes and generally more durable than seedling or all-stage resistance (Singh and Rajaram, 1992). On the basis of pooled ACI value $(<5.00)$, lines, DWRFB10, DWRFB12,
DWRFB14, DWRFB15, DWRFB19, DWRFB20, and DWRFB28 revealed highly resistant reaction to yellow rust across the locations at adult-plant stage (Table 3). The ACI value, in the range of $0-5$, is considered to possess strong seedling resistance instead of adult-plant resistance. Therefore, it appears that these lines might have strong all-stage resistance gene combined with few miner genes. The pooled ACI value of advanced breeding lines, DWRFB11, DWRFB13, DWRFB17, DWRFB18, DWRFB23, DWRFB27 and DWRFB29 
was in between 5 and 20. These lines supposed to possess high adult-plant resistance. None advanced line was categorised as susceptible with ACI $>60$. Earlier, 336 barley genotypes from ICARDA were evaluated against Indian Psh pathotype both at seedling and adult-plant stages. Of the total, 12 barley genotypes (ARAMIR/COSSACK, Astrix, C8806, C9430, CLE202, Gold, Gull, Isaria, Lechtaler, Piroline, Stirling, and Trumpf) were resistant to six Psh pathotypes (24, $57, \mathrm{M}, \mathrm{G}, \mathrm{Q}$ and $6 \mathrm{~S} 0$ ) both at the seedling and adultplant stages (Verma et al., 2018; Gyawali et al., 2018).

In conclusion, four advanced barley breeding lines, DWRFB12, DWRFB14, DWRFB19 and DWRFB20 were identified as nearly immune to yellow rust both at seedling and adult-plant stages. These lines have been developed by crossing indigenous and exotic genotypes and more adaptable to local environmental conditions. Therefore, identified resistant sources can be exploited directly or used in barley breeding program for developing yellow rust resistant varieties.

\section{Acknowledgement}

The authors convey their gratitude to different scientists and technical staff involved in the present study. The authors are also grateful to ICAR-IIWBR for providing financial support and facilities to conduct the research work.

\section{References}

1. Anonymous 2017. All India Coordinated Wheat and Barley Improvement Project Progress report 2016-17. ICAR-Indian Institute of Wheat and Barley Research, Karnal, India. 280p

2. Chen XM, RF Line and H Leung. 1995. Virulence and polymorphic DNA relationships of Puccinia striiformis f. sp. hordei to other rusts. Phytopathology 85(11): 1335-1342.

3. Duplessis S, CA Cuomo, YC Lin and A Aerts. 2011. Obligate biotrophy features unraveled by the genomic analysis of rust fungi. Proceedings National Academy of Sciences 108(22): 9166-9171.

4. Duveiller E, RP Singh and J Nicol. 2007. Challenges to maintaining wheat productivity: Pests, diseases and potential epidemics. Euphytica 157: 417-430.

5. Gangwar OP, S Kumar, P Prasad, SC Bhardwaj, $\mathrm{H}$ Khan and H Verma. 2016. Virulence pattern and emergence of new pathotypes in Puccinia striiformisf. sp. tritici during 2011-15 in India. Indian Phytopathology 69 (4s): 178-185.

6. Gangwar OP, SC Bhardwaj, GP Singh, P Prasad and S Kumar 2018. Barley diseases and their management: An Indian perspective. Wheat and Barley Research 10(3):138-150.

7. Gangwar OP, S Kumar, PL Kashyap, SC Bhardwaj, P Prasad, S Savadi, H Khan and Deepshikha. 2019. Virulence and molecular analysis of a typical pathotypes of yellow rust pathogen in India. Indian Phytopathology 72(2):187-194.

8. Gyawali S, RPS Verma, S Kumar, SC Bhardwaj, OP Gangwar, R Selvakumar, PS Shekhawat, S Rehman and Sharma-Poudyal D. 2018. Seedling and adult-plant stage resistance of a world collection of barley genotypes to stripe rust. Journal of Phytopathology 166(1):18-27.

9. Marshall D and RL Sutton. 1995. Epidemiology of stripe rust, virulence of Puccinia striiformis f.sp. hordei and yield loss in barley. Plant Disease 79: 732-737.

10. Nayar SK, M Prashar and SC Bhardwaj. 1997. Manual of current techniques in wheat rusts. Research Bulletin No. 2: Regional Station, Directorate of Wheat Research, Flowerdale, Shimla, India, 32p.

11. Pandey M, D Kopahnke, A Habekuss, W Friedt and F Ordon. 2009. Screening Nepalese hulless barley germplasm for resistance to major fungal and viral diseases. Journal of the Institute of Agriculture and Animal Science 30: 115-124

12. Park RF, HS Bariana and Wellings, CS. 2007. Preface. Australian Journal of Agricultural Research 58: 469.

13. Peterson RF, AB Campbell and AE Hannah. 1948. A diagrammatic scale for estimating rust intensity of leaves and stem of cereals. Candian Journal of Research Section 26: 496-500.

14. Prashar M, SC Bhardwaj, OP Gangwar and SK Jain. 2014. Virulence distribution and frequency of Puccinia striiformis f. sp. hordei, causing stripe rust of barley in northern parts of India. Indian Phytopathology 67 (1): 38-41.

15. Prakash V and RPS Verma. 2009. Inheritance of yellow rust resistance in barley (Hordeum vulgare L.). Indian Journal of Genetics and Plant Breeding 69(2): 99-101.

16. Sajid A, S Jawad, A Shah and H Rahman. 2009. Multi-location variability in Pakistan for partial resistance in wheat to Puccinia striiformisf. sp. tritici. Phytopathologia Mediterranea 48: 269-279.

17. Singh RP and S Rajaram 1992. Genetics of adult plant resistance to leaf rust in 'Frontana' and three CIMMYT wheats. Genome 35: 24-31. 
18. Stubbs RW, JM Prescott, EE Saari and HJ Dubin. 1986. Cereal Disease Methodology Manual. Mexico, D.F.: CIMMYT: 46p.

19. Vaish SS, SB Ahmed and K Prakash. 2011. First documentation on status of barley diseases from the high altitude cold arid Trans-Himalayan Ladakh region of India. Crop Protection 30: 1129-1137.

20. Verma RPS, V Kumar, B Sarkar, AS Kharub, D Kumar, R Selvakumar, R malik and I Sharma. 2012. Barley cultivars releases in India: names, parentages, origins and adaptations. Research bulletin no 29, DWR, Karnal, India, pp 26.
21. Verma RPS, R Selvakumar, OP Gangwar, PS Shekhawat, SC Bhardwaj, S. Rehman, D SharmaPoudyal and S Gyawali. 2018. Identification of additional sources of resistance to Puccinia striiformisf. sp. hordei (Psh) in a collection of barley genotypes adapted to the high input condition. Journal of Phytopathology 166(5): 355-364. 